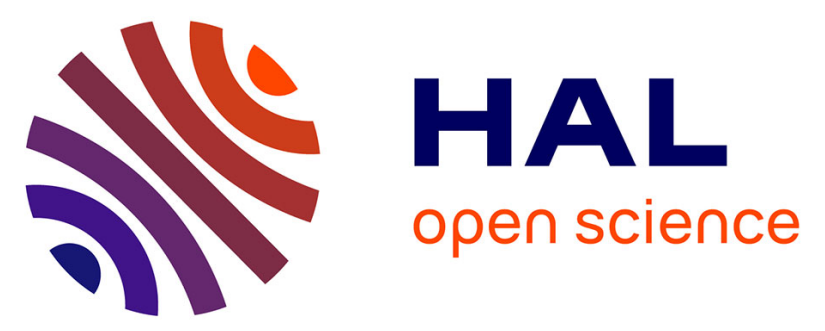

\title{
Effects of inhibitory neurons on the quorum percolation model and dynamical extension with the Brette-Gerstner model
}

\author{
Tanguy Fardet, Samuel Bottani, Stéphane Métens, Pascal Monceau
}

\section{To cite this version:}

Tanguy Fardet, Samuel Bottani, Stéphane Métens, Pascal Monceau. Effects of inhibitory neurons on the quorum percolation model and dynamical extension with the Brette-Gerstner model. Physica A: Statistical Mechanics and its Applications, 2018, 10.1016/j.physa.2018.02.002 . hal-01713016

\author{
HAL Id: hal-01713016 \\ https://hal.science/hal-01713016
}

Submitted on 20 Feb 2018

HAL is a multi-disciplinary open access archive for the deposit and dissemination of scientific research documents, whether they are published or not. The documents may come from teaching and research institutions in France or abroad, or from public or private research centers.
L'archive ouverte pluridisciplinaire HAL, est destinée au dépôt et à la diffusion de documents scientifiques de niveau recherche, publiés ou non, émanant des établissements d'enseignement et de recherche français ou étrangers, des laboratoires publics ou privés. 


\title{
Effects of inhibitory neurons on the Quorum Percolation model and dynamical extension with the Brette-Gerstner model
}

\author{
Tanguy Fardet ${ }^{\mathrm{a}, *}$, Samuel Bottani ${ }^{\mathrm{a}}$, Stéphane Métens ${ }^{\mathrm{a}}$, Pascal Monceau ${ }^{\mathrm{a}, \mathrm{b}}$ \\ ${ }^{a}$ Laboratoire Matière et Systèmes Complexes, UMR 7057 CNRS, Université Denis \\ Diderot-Paris 7, 10 rue A. Domon et L. Duquet, 75013 Paris Cedex, France. \\ ${ }^{b}$ Université d'Evry-Val d'Essonne, France.
}

\begin{abstract}
The Quorum Percolation model (QP) has been designed in the context of neurobiology to describe the initiation of activity bursts occurring in neuronal cultures from the point of view of statistical physics rather than from a dynamical synchronization approach. This paper aims at investigating an extension of the original QP model by taking into account the presence of inhibitory neurons in the cultures (IQP model). The first part of this paper is focused on an equivalence between the presence of inhibitory neurons and a reduction of the network connectivity. By relying on a simple topological argument, we show that the mean activation behavior of networks containing a fraction $\eta$ of inhibitory neurons can be mapped onto purely excitatory networks with an appropriately modified wiring, provided that $\eta$ remains in the range usually observed in neuronal cultures, namely $\eta \lesssim 20 \%$. As a striking result, we show that such a mapping enables to predict the evolution of the critical point of the IQP model with the fraction of inhibitory neurons. In a second part, we bridge the gap between the description of bursts in the framework of percolation and the temporal description of neural networks activity by showing how dynamical simulations of bursts with an adaptive exponential integrate-and-fire model lead to a mean description of bursts activation which is captured by Quorum Percolation.
\end{abstract}

\footnotetext{
${ }^{*}$ Corresponding author

Email address: tanguy.fardet@univ-paris-diderot.fr (Tanguy Fardet)
} 
Keywords: quorum percolation, inhibitory neurons, neuronal cultures, dynamical model

\section{Introduction}

Neuronal rhythms are widespread oscillating phenomena, both in vivo and in vitro, which were observed over many temporal scales [1. Hitherto, the fundamental mechanisms underlying their occurrence is far from being fully understood and is the subject of a significant research activity; it involves several scientific fields, from fundamental biology, information theory [2], physics of dynamical systems and critical phenomena [3] to graph topology [4] and massive parallel computation [5, 6]. The human brain is a very complex network, with about $10^{11}$ neurons [7, each of them connected to 1000-15000 others. Moreover, it is organized in localized computational units connected according to a well defined hierarchical structure. Thus, although investigation and imaging techniques enabling to record the cerebral activity in vivo are making significant progress [8, 9], the mere size and complexity of the brain makes its whole description and understanding a far-sighted goal. Complementary to observations 15 and experiments on real brains, in vitro experiments on dissociated neuronal cultures are an invaluable tool in investigating the fundamental questions on neuronal dynamics set above. Such cultures are usually obtained by seeding dissociated neurons extracted from rodent embryos, or alternatively neuronal stem cells, on a suitable substrate. Though similar monitoring can be performed on brain slices, we will focus on the activity of dissociated cultures, where axons and dendrites grow in such a way that neurons self-organize after a few days into a two-dimensional network exhibiting a high level of randomness [10]. As a matter of fact the connectivity between neurons is described by probability distributions. These neuronal cultures hold between $10^{3}$ and $10^{5}$ neurons with typical densities between 500 and 5000 neurons per $\mathrm{mm}^{2}$, each of them connected via a number of synapses falling between 20 and 200 [11. These changes in connectivity and scale compared to a brain could, at first glance, appear as 
a loss from a neurobiologic point of view; yet, they are a key feature for the complementary approach of in vitro experimentation to study neuronal activity and growth. Quantitative measurements of the neural activity inaccessible in vivo can be carried out with the help of micro-electrode arrays (MEA) [12, optogenetics, and calcium imaging [13].

Synchronized periodic bursts of spiking activity have been regularly observed in dissociated neuronal cultures [14, 15] and appear as a fundamental emergent spatio-temporal property of neuronal populations. Bursts of activity can also be artificially triggered by externally activating a fraction of neurons. The Quorum Percolation model (QP) has been elaborated to describe the initiation of bursts observed in such cultures as a collective phenomenon, from the point of view of statistical physics rather than dynamical systems [16]. Under its original form 40 the QP model does not take into account the presence of inhibitory neurons. However, a general description of collective behaviors in neural networks requires the integration of inhibitory neurons in the QP model, since it has been pointed out that they can play a role in the structure of bursts [17, 18. We devoted recently several studies to extend the original QP model by including additional biological relevant properties and modulation of the neuronal activity: the decay of the neuronal voltage accounting for ions leakage through the neuron membrane [19], variability in the quorum accounting for a modulation of the neuronal excitability threshold [20], finite size scaling and the derivation of a normal form around the critical point together with a preliminary study of the incorporation of inhibitory neurons 21. In this last paper, we suggested that under specific conditions, the mean characteristics of the burst activation of networks with inhibitory neurons are the same as the ones of purely excitatory networks with different effective connectivity. The first goal of this paper is to provide a deeper investigation of the mapping between the presence of inhibitory neurons and an equivalent purely excitatory reduced connectivity. We point out what should be learned from the mean field approach, we characterize the connectivity features of the purely excitatory network accounting for a fraction of inhibitory neurons, we quantify its equivalence domain and derive a 
relation between the critical point and the fraction of inhibitory neurons. As inhibitory neurons are commonly assumed to play a modulating role of neuronal activity and spatio-temporal coordination, we investigate the validity of our previous conclusions in a dynamical setting. Thus, the second goal of this paper is to show that the key features of Quorum Percolation captured by the simple, discrete model with inhibition are preserved in a fully dynamical model based on biologically more refined description of neurons and synapses, namely the adaptive Exponential Integrate-and-Fire model [22. However, it should be noticed that the dynamics of the activity cannot be captured by IQP and QP models, since they deal with equilibrium properties of the short time onset of bursts.

\section{The original Quorum Percolation model}

The original Quorum Percolation (QP) model is a discrete-time cellular automaton describing the propagation of information on a graph through a minimal set of rules for activation cascades in neuronal populations. Since neuronal communication through synapses is directional, the neuronal population

75 is represented by a directed graph connecting neurons located on the vertices. Specifically introduced to describe the onset of activity bursts observed in small, in vitro cultures [16, the model is based on a non spatial graph considering only the node connectivities and constructed by randomly choosing, for each neuron $i, k$ incoming links among the $N-1$ other neurons according to an in-degree so probability distribution $p_{k}$. It is worth noticing that such a random description of the incoming links probability relevant in the case of cultures of dissociated neurons grown in an in vitro environment does not work anymore in the case of neuronal cultures that have grown in vivo like brain slices or animal visual cortex [23].

In the QP model, each neuron $i$ is represented by a discrete variable $V_{i}(t)$ which accounts for the membrane potential, and by a neuronal state - at rest or active - with activation governed by a threshold rule. A neuron is activated 
between $t-\Delta t$ and $t$ if its potential becomes greater or equal to some activation threshold $m$; once activated, it sends signals to its outgoing neighbors. As the models represents only one activation wave, an activated neuron remains so and sends no further signals in the following steps. After a time step $\Delta t$, each neuron $i$ integrates the signals it received by incrementing its potential $V_{i}(t-\Delta t)$ by the sum of the inputs from its incoming neighbors activated during the elapsed time interval. All the signals are taken identical and associated to an integer increment equal to +1 , which sets the scale for the threshold value $m$. The network is stimulated at time $t=0$ by an initial excitation of the network, performed by activating a given fraction $f$ of randomly chosen neurons.

The activity of the network at time $t$ is given by the fraction of active neurons $\phi(t)$, increasing with $t$, and converging towards a stationary value $\Phi(f, m)$ after a few time steps, dependent on the initial active fraction $f$ and the threshold $m$. As first reported by Cohen et al. [16] the surface $\Phi(f, m)$ (noted simply $\Phi$ in the following) defines a phase diagram as shown on Fig. 1, where two regimes can be distinguished depending on $m$. Below some critical value $m_{c}, \Phi$ presents a discontinuity at some value $f^{*}(m)$ when the control parameter $f$ is varied, whereas it remains continuous above $m_{c}$. The sudden jump occurring at $f^{*}(m)$ is associated with a percolation phenomenon on the network, where a very small variation of $f$ results in the appearance of a giant cluster, whose normalized size is given by the difference between the lower and upper values of $\Phi$ at the discontinuity. Despite its simplicity the phase diagram of QP model captures the key behavior observed in experiments in the group of E. Moses [11, 16] on induction of activity in neuronal cultures which exhibits the same emergence of a giant cluster depending on neuronal excitability. Following the usual concepts of percolation on lattices 24] for the second order transition between the presence and absence of a percolation phenomenon, the amplitude of the jump $\langle g\rangle$ is considered as the standard order parameter, whose behavior in the vicinity of $m_{c}$ follows a power law:

$$
\langle g\rangle \sim\left(\frac{m_{c}-m}{m_{c}}\right)^{\beta} .
$$




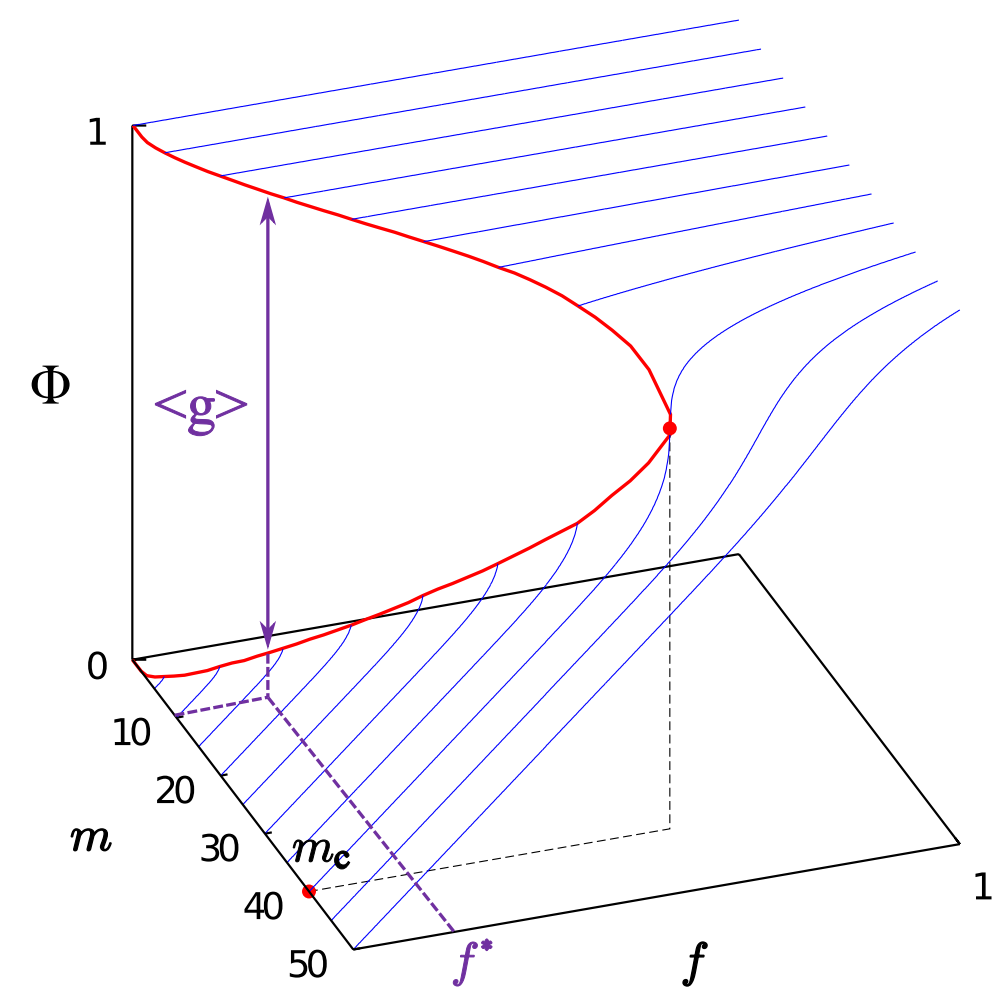

Figure 1: Phase diagram of the Quorum Percolation model, for a Gaussian in-degree distribution with a mean $\bar{k}=50$ and a standard deviation $\sigma_{k}=10$. When the quorum $m$ is smaller than $m_{c}$, a jump in the fraction of active neurons $\Phi$ occurs when increasing the fraction $f$ of initially activated neurons from zero. The height $\langle g\rangle$ of the jump at the discontinuity is the normalized size of the giant percolation cluster.

Since $m$ is discrete, it is difficult to extract a precise critical value of $\beta$ from the behavior of $\langle g\rangle$, mainly because the uncertainty over the value of $m_{c}$ is at least of 1 unit. We overcame such an hurdle by carrying out an extension of the quorum percolation mean-field theory to non integer values of $m$ and we showed [25] that a Gaussian distribution $p_{k}$ of incoming links leads to $m_{c}=$ $\bar{k}\left(1-a\left(\frac{\sigma}{\bar{k}}\right)+b\left(\frac{\sigma}{\bar{k}}\right)^{2}\right)$ where $\bar{k}$ is the mean value of the number of incoming links and $\sigma$ the width of $p_{k}$; for values of $\bar{k}$ lying in the range of experiments on mature cultures, we found that $a \in[1.27,1.30]$ and $b \in[1.56,1.59]$. Moreover we showed that the value of $\beta$ for such incoming links distributions is compatible 
with $\beta=1 / 2$.

Defined as above the model is only related to the topological relationships between nodes and does not take into account spatial properties from localization of neurons is space. Hence, no metric is involved and the percolation cannot be described with respect to a given dimensionality as in the usual case of lattices [24]. As showed by Tlusty and Eckmann [26] for small dense neuronal cultures as those used for the global activation experiments, the spatial embedded neuronal network is in practice a fully randomly connected one. For large cultures, as for instance those investigated by Orlandi et al. 27] activity propagation fronts are observed and a spatial metric has to be considered for the study of the neuronal culture dynamics. Furthermore, it has recently been shown on the basis of another statistical physics model of neuronal cultures, the random field Ising model, that metric correlations induce strong deviations from the mean field 28 .

\section{A quorum percolation model with inhibitory neurons: IQP}

\subsection{Main features of the IQP model and comparison with experiments}

Let us now assume that a fraction $\eta$ of neurons, drawn at random, is inhibitory. As in the original model, the network is wired in such a way that, for each neuron, the number of incoming links follows a probability distribution $p_{k}$; however, we now set every outgoing link of an inhibitory neuron to "inhibitory". We account for these neurons in the following way: when an inhibitory neuron fires, its sends a signal equal to -1 instead of +1 through its outgoing links, thus decrementing the potential of each target. Hence, a neuron becomes active if the number of its active excitatory incoming neighbors $e$ minus the number $i$ of its active inhibitory ones is greater than the quorum: $(e-i) \geq m$. A sketch explaining the progress of the Quorum Percolation with inhibitory neurons (IQP) is provided in Fig. 2. It should be noticed that, unlike the QP model, the potential of a neuron is no more a monotonous increasing function of the time

${ }_{135} t$ associated with the discrete time kinetics, but the fraction of active neurons 
(inhibitory and excitatory) necessarily increases with $t$ because of the threshold rule. Running Monte-Carlo simulations to compute the stationary activity $\Phi$ involves (i) constructing a random network $\mathcal{G}$ of $N$ neurons according to the incoming links probability distribution $p_{k}$, (ii) declaring a fraction $\eta$ of neurons inhibitory according to an uniform random distribution, (iii) activating a fraction $f$ of the neurons regardless of their excitatory or inhibitory nature and (iv) processing the quorum activation rule until the number of active neurons stops increasing.

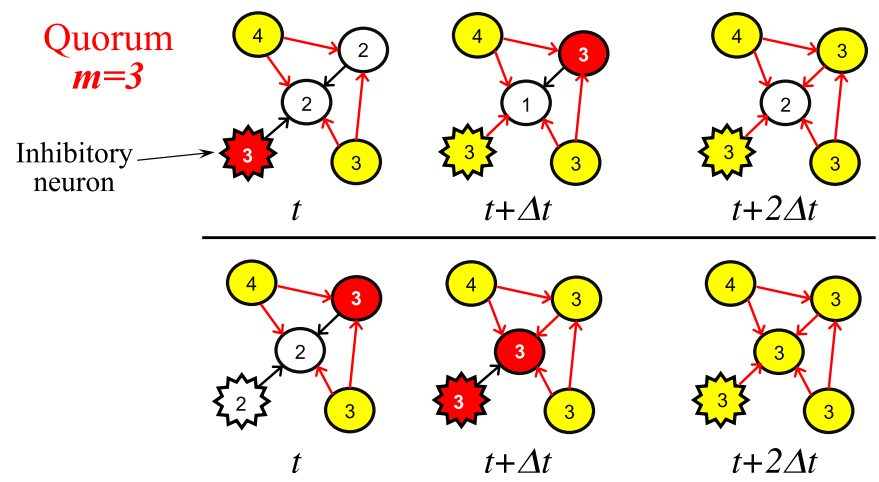

Figure 2: Arrows represent the directed axonal links between neurons. The neurons associated with the light grey (yellow) color are active (i.e. they already fired) while the white ones are at rest and the red ones are just firing at the indicated time. One inhibitory neuron is represented as a dented circle. Upper figure: at time $t$, the inhibitory neuron fires (because of external inputs which are not represented here); thus the potential of its outgoing neighbor, in the center, shifts from 2 to 1 . Let us suppose that, at time $t+\Delta t$ the upper right neuron fires (also because of external inputs not represented here); the potential of its outgoing neighbor is incremented by one, leading to the state represented at $t+2 \Delta t$. The bottom figures show that the order in which the neurons fire matters in the presence of inhibitory neurons. If we assume that the right upper corner excitatory neuron fires before the left down inhibitory one (because of another history of external inputs than on the first row) the central neuron now activates, while it will never fire before the end of the process in the example above.

We carried out explicit Monte Carlo simulations of the IQP model for Gaussian incoming links distributions involving 100000 neurons, four values of $\bar{k} \in\{25,50,75,100\}$, ten values of $\eta$ ranging from 0.05 to 0.2 , and three different values of $\sigma$ in each case; these ranges were chosen to be consistent with 
the experimental estimations [11, 16]; in each case, the value of $\Phi$ as a function of $f$ was averaged over 29 different network configurations. A selection of some of these explicit IQP simulations are shown as solid red lines in Fig. 3 to Fig. 4 for networks with different mean connectivity as a function of $\eta$.

Our main result is that, for $\eta$ under 25 percent (i.e. less than a quarter of the whole population is inhibitory), the presence of the inhibitory neurons does not change the qualitative behavior of the quorum percolation phase diagram: for a given value of $\eta$, jumps in the activity will occur, provided that $m$ is smaller than a critical value $m_{c}$, which depends on $\eta$.

Furthermore, Fig. 5 shows the influence of $\eta$ for a fixed value of the threshold $m$ and fixed values of the connectivity parameters $\{\bar{k}, \sigma\}$. Indeed, when the ratio $\eta$ of inhibitory neurons in increased, the position $f^{*}$ of the jump in $\Phi$ is shifted towards greater values of the initial activity, while its size $g$ is decreased (until it possibly vanishes). Hence, when inhibition increases for a given firing threshold and a given connectivity, a more important fraction of initially activated neurons is necessary to trigger the percolation.

Communication between neurons involves chemical signaling at the synaptic level: neurotransmitters present in the pre-synaptic domain are released by vesicle exocytosis and bind to receptors located in the post-synaptic domain. This release is triggered by electrical signaling conveyed by action potentials, and is the biological equivalent of the update of a node's potential by its active neighbours. In neuronal networks, both excitatory and inhibitory synapses are present, the latter being associated to $G A B A_{A}$ (Gamma-aminobutyric acid) receptors. These receptors can be hindered, and even blocked by adding specific drugs. Using bicuculline in the culture medium to block $G A B A_{A}$ receptors Soriano et al. 14 compared the activations of fully excitatory networks and of untreated mixed excitatory and inhibitory cultures and observed that the 175 presence of inhibitory neurons decreases the threshold for the percolation phenomenon compared to the purely excitatory case. Such an experimental result is very well described in the framework of the IQP model and predicted by our simulations. 

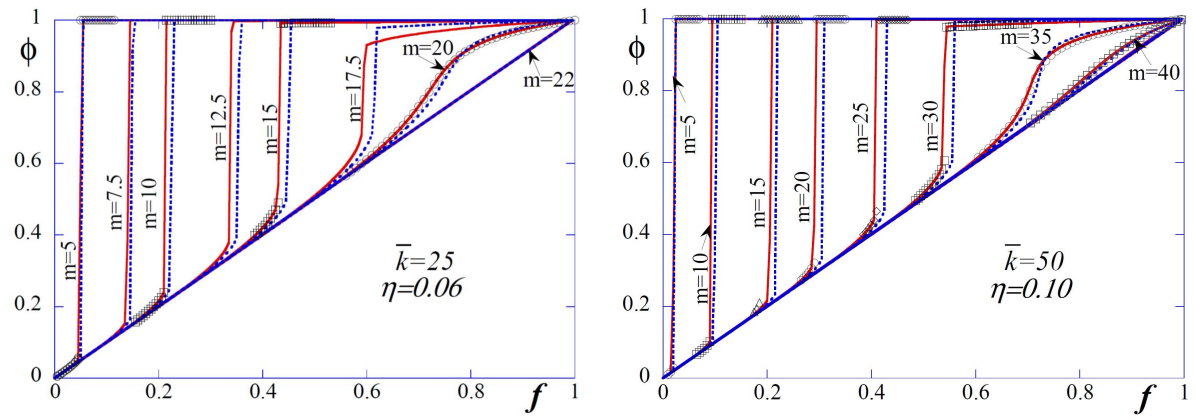

Figure 3: Comparison between explicit IQP Monte Carlo simulations (solid red lines), solutions of the mean-field equation around the jumps (open black symbols), and QP simulations of the equivalent purely excitatory network obtained trough $\bar{k}_{e q}=\bar{k}(1-2 \eta)$ (blue dotted lines). $\bar{k}=25, \sigma=2.5$ and $\eta=0.06$ (left) $\bar{k}=50, \sigma=5$ and $\eta=0.10$ (right).
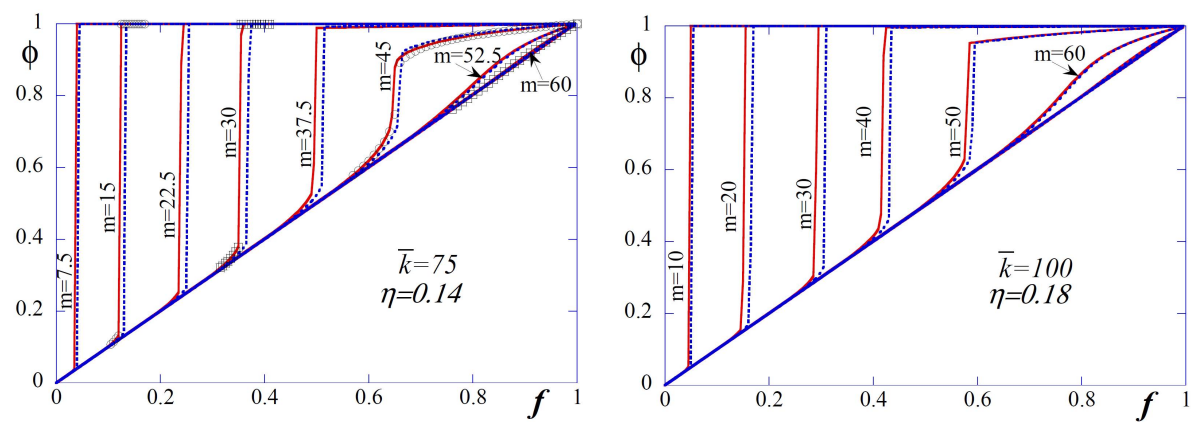

Figure 4: Comparison between explicit IQP Monte Carlo simulations (solid red lines), solutions of the mean-field equation around the jumps (open black symbols), and QP simulations of the equivalent purely excitatory network obtained trough $\bar{k}_{e q}=\bar{k}(1-2 \eta)$ (blue dotted lines). $\bar{k}=75, \sigma=7.5$ and $\eta=0.14$ (left) $\bar{k}=100, \sigma=10$ and $\eta=0.18$ (right).

\subsection{Mean-field theory}

An alternative approach for calculating the stationary fraction of active neurons $\Phi$ can be deduced from a mean-field leading to a self-consistency equation 21. Indeed $\Phi$ is also the probability for a neuron to be active at equilibrium and it corresponds to the probability to be either active through initial stimulation or to be activated during the IQP discrete signal propagation process. This activation probability of a neuron in the cascade depends itself upon $\Phi$ and can be approximated by binomial processes given $m$ and $p_{k}$. In order to obtain 


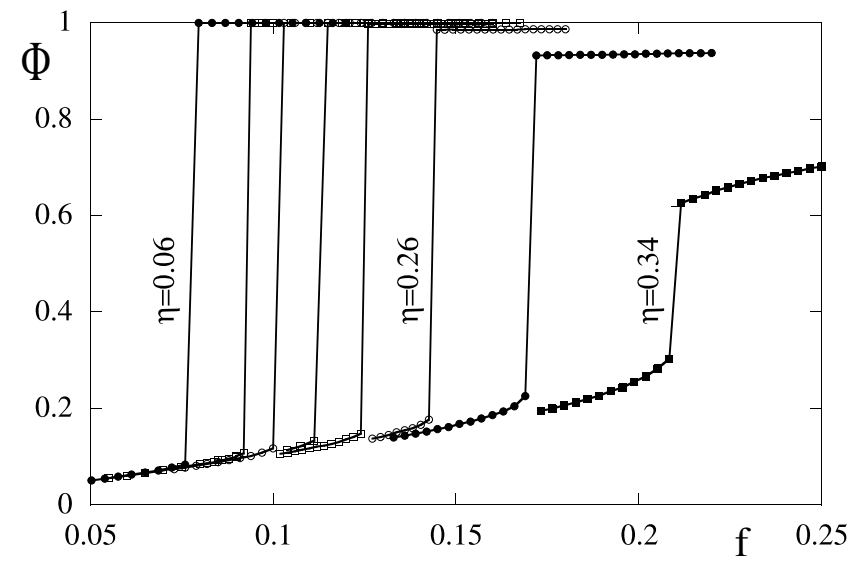

Figure 5: Evolution of the jump in the activity when the ratio $\eta$ of inhibitory neurons increases from 0.06 (leftmost jump) to 0.34 (rightmost jump) in steps of 0.04 . These values are obtained via the mean-field equation, for a fixed threshold $m=10$ and fixed parameters $\{\bar{k}=50, \sigma=$ $0.05\}$ for the network connectivity.

this self-consistency equation, we first partition the set of neurons according to their number $k$ of incoming links and consider a neuron of the network with $k_{e}$ excitatory and $k_{i}$ inhibitory incoming links ( $k$ is fixed). The probabilities that $Y=e$ excitatory neurons among $k_{e}$ and $Z=i$ inhibitory ones among $k_{i}$ are active read respectively $\mathcal{P}_{e x}(Y=e)=\left(\begin{array}{c}k_{e} \\ e\end{array}\right) \Phi^{e}(1-\Phi)^{k_{e}-e}$ and $\mathcal{P}_{i n}(Z=i)=$ $\left(\begin{array}{c}k_{i} \\ i\end{array}\right) \Phi^{i}(1-\Phi)^{k_{i}-i}$. The probability for the target neuron to exceed the quorum ( to have $e-i \geq m$ ) can be calculated by noticing that an activation occurs if $Z=i$ only if $Y \geq m+i$. Hence the activation probability of this neuron can be written as the double sum: $\mathcal{P}(e-i \geq m)=\sum_{i=0}^{k_{i}} \mathcal{P}_{i n}(Z=i) \sum_{e=m+i}^{k_{e}} \mathcal{P}_{e x}(Y=e)$. Now, the probability that a neuron with $k$ incoming links has $k_{i}$ inhibitory ones reads $P\left(k_{i} \mid k\right)=\left(\begin{array}{c}k \\ k_{i}\end{array}\right) \eta^{k_{i}}(1-\eta)^{k-k_{i}}$, assuming that $\eta$ is also the fraction of inhibitory incoming links, as we will discuss further. In the end, the self 
consistency equation can be written:

$$
\begin{aligned}
\Phi=f+(1-f) & \sum_{k=m}^{\infty} p_{k} \sum_{k_{i}=0}^{k-m}\left(\begin{array}{c}
k \\
k_{i}
\end{array}\right) \eta^{k_{i}}(1-\eta)^{k-k_{i}} \\
& \sum_{i=0}^{k_{i}}\left(\begin{array}{c}
k_{i} \\
i
\end{array}\right) \Phi^{i}(1-\Phi)^{k_{i}-i} \sum_{e=m+i}^{k-k_{i}}\left(\begin{array}{c}
k-k_{i} \\
e
\end{array}\right) \Phi^{e}(1-\Phi)^{k-k_{i}-e} .
\end{aligned}
$$

180

We compared the results of our Monte-Carlo simulations for 100000 neurons with the values of $\Phi$ provided by the resolution of equation (2) focused on the vicinity of the jumps, in some cases showed in Fig. 3 and Fig. 4 . This extension of the range of our investigation with respect to [21] where only 10000 neurons populations were simulated shows in a robust way a very good agreement between the two approaches; we checked that this agreement increases with the size of the network because of finite size effects, since the mean-field approach is expected to hold in the infinite limit. The agreement is remarkable as the mean field approach is not designed to take into account temporal correlations while strictly speaking, the actual IQP process is sensitive to them. Indeed, Fig.

1902 shows that the order in which a neuron receives signals can come into play whereas it does not matter in the absence of inhibitory neurons. Nevertheless, a reason why the mean field actually works is that the order of activation hardly comes into play in the information propagation process but when the state of the neurons are close to firing, that is just below the quorum.

3.3. Mapping of the IQP model on purely excitatory networks

A close look at the IQP rules suggests that a neuron with $k$ incoming links, $k_{i}$ of them being inhibitory, could in average behave as a neuron with $k-2 k_{i}$ purely excitatory incoming links: each inhibitory neuron can be viewed as canceling one of the excitatory. This can be for example observed for the central neuron in the sketch of the upper row of Fig. 2 starting from a value of its potential equal to 2 , it ends with the same value since the inhibitory and excitatory inputs compensate each other: it is as if the links with the left down and the upper right neurons had been erased. The robustness of the agreement of 
the IQP mean-field theory with Monte-Carlo simulations suggests that such an

a mixed excitatory and inhibitory network with a mean number $\bar{k}_{i}$ of inhibitory incoming links and $\bar{k}-\bar{k}_{i}$ excitatory ones should lead to the same stationary state as a purely excitatory network with $\bar{k}-2 \bar{k}_{i}$ mean incoming links. In order to check this hypothesis, we ran additional Monte-Carlo simulations: Assuming that $\overline{k_{i}}=\eta \bar{k}$, we simulated for each set $\{\bar{k}, \sigma, \eta, m\}$ already investigated in the framework of the IQP model, an associated QP set $\left\{\bar{k}_{e q}=\bar{k}(1-2 \eta), \sigma, m\right\}$ without inhibitory neurons. Some typical results are reported in Fig. 3 and Fig. 4. where comparisons of the activity computed by the two processes are shown. As shown on these figures, the stationary response of the mixed excitatory and inhibitory networks to a given external excitation $f$ is indeed remarkably close to the one of the associated purely excitatory network with the equivalent reduced number of incoming links. From these figures, we can notice that the differences between the two approaches depend on $m$ and $f$ : They are more pronounced when $m$ increases and in the vicinity of the jump, where the percolation process makes the fraction $\Phi$ of active neurons undergo a steep variation, from a value just above $f$ to a value close to 1 . However a quantitative analysis of these differences can be achieved from a global point of view by computing a (renormalized) Minkowski distance between the IQP and the associated QP response over the whole excitation range as:

$$
\Delta=\frac{1}{N} \sqrt{\sum_{i=1}^{i=N}\left(\Phi\left(f_{i}\right)-\Phi_{e q}\left(f_{i}\right)\right)^{2}}
$$

where a subscript $i$ must be added to the initial value of the excitation parametrized by $f$ to define properly $\Delta ; f_{i}$ runs from 0 to 1 , and $i$ from 1 to $N=$ 200. $\Phi\left(f_{i}\right)$ and $\Phi_{e q}\left(f_{i}\right)$ denote respectively the original IQP activity and the QP activity on equivalent excitatory networks averaged over 29 configurations, as responses to an excitation parametrized by $f_{i}$; since $\Phi\left(f_{i}\right) \in[0,1], \Delta$ lies between zero for identical global responses and one for maximal disagreeing responses. Fig 6 and Fig. 7 show the evolution of $\Delta$ in the $\eta-m$ plane. These results call 
for the following comments: obviously, if $m$ is greater than $k_{e q}$ the differences between IQP and QP are negligible because the activation probability are very low, since $\Phi$ and $\Phi_{e q}$ are very close to $f$ all over the range $[0,1]$. For a given value of $\eta$ the differences between the two approaches exhibit a maximum at an intermediate value of the quorum, while for a given value of $m$, the difference increases with $\eta$, excepted if $m$ is too low. Lastly the meaningful scale of the discrepancies decreases as the mean connectivity $\bar{k}$ of the network increases.
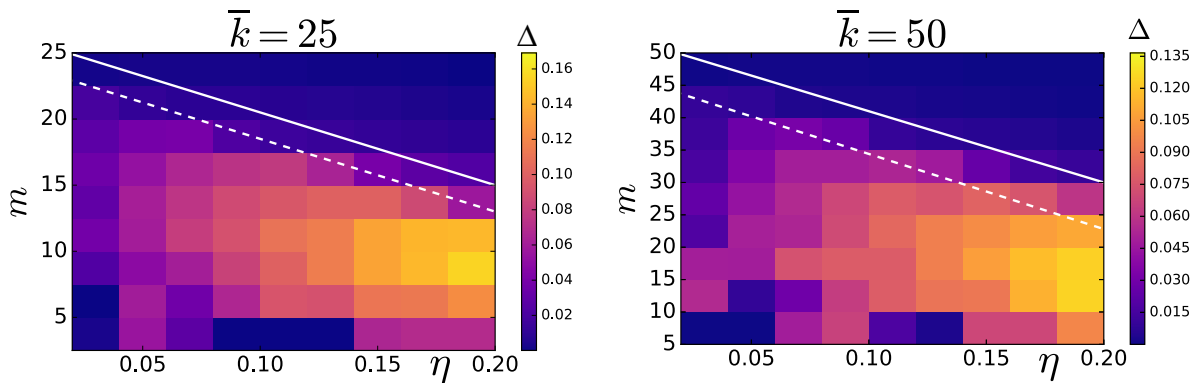

Figure 6: Minkovski distances $\Delta$ between the IQP model and the equivalent QP model without inhibitory neurons as a function of $m$ and $\eta$ for $\bar{k}=25$ and $\bar{k}=50$. The solid white lines represent $k_{e q}$ and the dotted ones $m_{c}$ as a function of $\eta$.
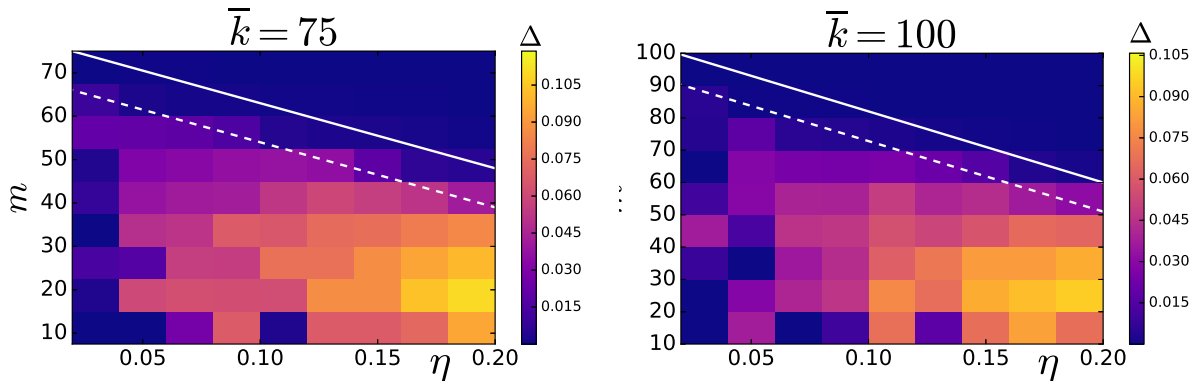

Figure 7: Minkovski distances $\Delta$ between the IQP model and the equivalent QP model without inhibitory neurons as a function of $m$ and $\eta$ for $\bar{k}=75, \sigma=7.5$ and $\bar{k}=100, \sigma=10$. The solid white lines represent $k_{e q}$ and the dotted ones $m_{c}$ as a function of $\eta$.

Besides the global comparison of the QP and IQP model, we carried out a local analysis by investigating a physical quantity describing the critical behavior: the order parameter. Typical results are shown in Fig. 8 where values of 
the order parameter (averaged over 29 configurations in each case) calculated from simulations of the IQP model are compared with values extracted from the equivalent QP model. As long as $\eta$ is below 10\%, the mean relative differences in the two approaches $\left\langle\frac{\delta g}{g}\right\rangle$ remain below $7 \%$. We can retrieve a slight increase in the differences and in the relative differences $\left\langle\frac{\delta g}{g}\right\rangle$ as $\eta$ is increased and an increasing agreement as $\bar{k}$ increases. It should be noticed that these results take into account uncertainties on the calculation of $\langle g\rangle$; these uncertainties grow very quickly when getting close to the critical point and the comparisons are no more meaningful. As a matter of fact, when linking these results with the ones set out in the last subsection, we can conclude that an important part of the differences in Minkowski distances (Fig. 6] and Fig. 7) stems from the shift in the position of the jump rather than its height.

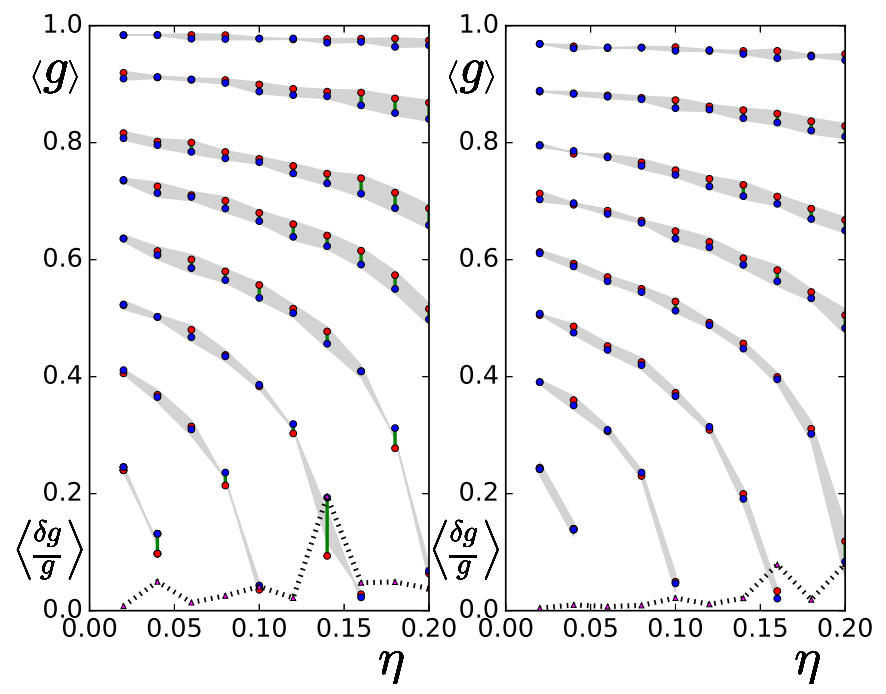

Figure 8: Evolution of the order parameter $\langle g\rangle$ calculated from the IQP model (red circles) and the equivalent $\mathrm{QP}$ one (blue circles) as a function of $\eta$ in the cases where $\bar{k}=50$ (left) and $\bar{k}=100$ (right) for 8 different values of $m$ ranging from $0.9 \bar{k}$ to $0.2 \bar{k}$ from bottom to top. The shaded area accounts for the differences between the two approaches and the dotted line follows the mean relative deviations between them for different values of $\eta$. 


\subsection{Critical point of the IQP model}

255 of 1 unit in $m$ in order to estimate the critical values $m_{c}(\eta)$ of the quorum as a function of $\eta$ for the four different values of the mean incoming links numbers already investigated. We were able to estimate $m_{c}(\eta)$ within an uncertainty of 1 unit for $\bar{k}$ equal to 25 and 50 and an uncertainty of 2 units for $\bar{k}$ equal to 75 and made fully excitatory by addition of bicuculline in the culture and $m_{c E I}$ for the mixed network are linked by the approximated relation $\frac{m_{c E I}}{m_{c E}}=1-\frac{\bar{k}_{I}}{\bar{k}_{E}}$ where $\bar{k}_{E}$ and $\bar{k}_{I}$ designate the mean numbers of excitatory and inhibitory incoming links of the mixed network.

where $m_{c}(\eta=0)=(\bar{k}-a \sigma)$. Results of the fits of the lines observed on Fig. 9 are set out in Table I; as a main result, the evolution of the critical point $m_{c}(\eta)$ with the fraction of inhibitory neurons extracted from IQP simulations is predicted by the QP theory applied to the equivalent network with a remarkable agreement. $m_{c}(\eta)$ gives a relation between the two parameters characterizing the the total distribution of incoming links (excitatory and inhibitory) $\{\bar{k}, \sigma\}$ and the fraction of inhibitory neurons. Let us notice that it is a little bit different from the results obtained by Soriano et al. [14] in a situation where $p_{k}$ is Poissonian: they showed that that the critical values $m_{c E}$ for a mixed network 


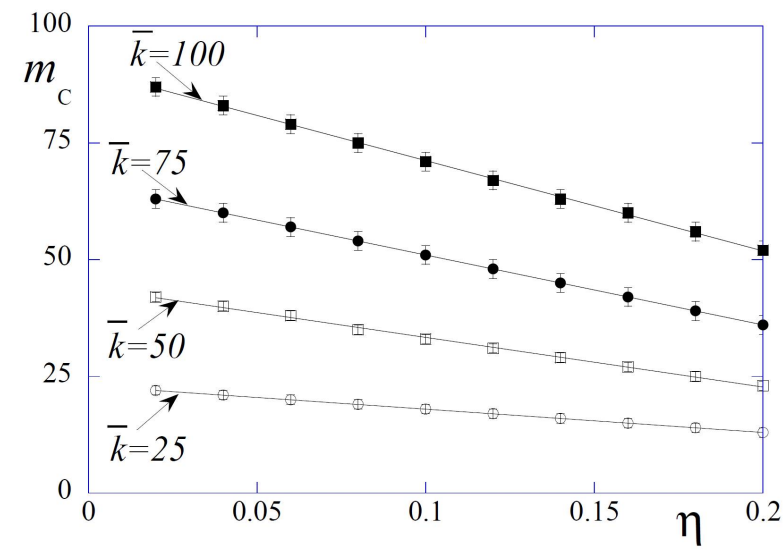

Figure 9: Evolution of the critical point $m_{c}$ as a function of $\eta$ for the four different values of $\bar{k}$ investigated with $\sigma=0.1 \bar{k}$

\begin{tabular}{|c|c|c|}
\hline $\bar{k}$ & $m_{c}(\eta=0)$ & $m_{c}(\eta)(\mathrm{fits})$ \\
\hline 25 & 21.7 & $23-50 \eta$ \\
\hline 50 & 44.3 & $44-106 \eta$ \\
\hline 75 & 64.4 & $66-150 \eta$ \\
\hline 100 & 88.8 & $90-194 \eta$ \\
\hline
\end{tabular}

Table 1: Results of the fits of the four straight lines represented on Fig. 9. values of the critical points calculated from the continous extension of the QP model are recalled in the middle column of the table

\section{Validity of the quorum percolation paradigm in a dynamical frame-} work

As percolation is sufficient to describe the initiation of bursts, it should be investigated if properties of the basic theoretical percolation models remain valid in more realistic situations. As mentioned previously, percolation phenomena, with and without inhibition, have been experimentally validated by the match of the original minimal model to the experimental observations on neuronal cultures by the group of E. Moses [11, 14, 16]. This significant evidence of 
quorum percolation phenomenon in living neuronal networks concerned however only the single response of a neuronal population to an external activation signal of increasing strength. Although it would be surprising for a percolation process to happen only in this circumstance, its occurrence during the longterm activity of a neuronal culture remains to be characterized. This question is specifically relevant when focusing on networks with inhibitory neurons, as inhibition plays a role on the temporal correlations of neuronal activity in a population. Thus, this last section is devoted to the investigation of quorum percolation in the framework of a dynamical model of neuronal networks. We show on a generic example that the percolation description remains relevant to describe the initiation of a burst of activity inside a population of dynamical neurons, then discuss how this phenomenon can also be observed in simulations of spontaneous neuronal activity.

Similarly as for the IQP model, we use and generate a random network $\mathcal{G}$ with Gaussian distribution $p_{k}$ of incoming links characterized by $\bar{k}$ and $\sigma$. Each node is now described by a differential equation model that realistically describes the membrane potential variation in time and spikes. The input links are represented by terms in the neuronal state differential equation that describe the positive (for excitation) or negative (for inhibition) time varying post-synaptic potential of synpases. We chose here the adaptive Exponential Integrate-andFire (aEIF) model [22] because of its compromise between simplicity and biological relevance. In this model, the dynamical evolution of a neuron is described by two variables - its membrane potential $V$ and a slow adaptation current $w$ - which are governed by the following equations:

$$
\begin{aligned}
& \left\{\begin{array}{l}
C_{m} \frac{d V}{d t}=-g_{L}\left(V-E_{L}\right)+g_{L} \Delta_{T} \exp \left(\frac{V-V_{t h}}{\Delta_{T}}\right)-w+I \\
\tau_{w} \frac{d w}{d t}=a\left(V-E_{L}\right)-w
\end{array}\right. \\
& \text { if } V>V_{\text {peak }}\left\{\begin{array}{l}
V \leftarrow V_{r} \\
w \leftarrow w+b
\end{array}\right.
\end{aligned}
$$

all neuronal parameters are defined in [22]. Hence we will only mention the two most relevant in this study, which are $E_{L}$, the resting potential of an isolated 
neuron, and $V_{t h}$, the "threshold" potential, which marks the beginning of a spike initiation, generated by the diverging exponential. The difference $V_{t h}-$ $E_{L}$ is therefore closely related to the quorum defined in the IQP model. The connection from any neuron $A$ to a second neuron $B$ is implemented using alphashaped post-synaptic currents (PSCs) in the input term $I$ which leads, if a spike occurs at $t=0$, to a subsequent current of the form

$$
I_{s}(t)=s_{A B} \frac{I_{0}}{\tau_{s}} t e^{-t / \tau_{s}}
$$

where $s_{A B}$ is the dimensionless synaptic strength and $I_{0}=e \cdot 1 p A$ is a nortimestep, i.e. $0.1 \mathrm{~ms}$. For this first part, the units are all implemented with parameters for adaptive spiking neurons, though this has no significant impact on the involved timescale. 


\begin{tabular}{ccc}
\hline Parameters & $\boldsymbol{f}^{*}(\mathbf{I Q P})$ & $\boldsymbol{f}^{*}$ (Sim.) \\
\hline $\bar{k}=25, m=5$ & 0.05 & {$[0.06 ; 0.08]$} \\
$\bar{k}=25, m=10$ & 0.18 & {$[0.19 ; 0.21]$} \\
$\bar{k}=25, m=15$ & 0.45 & $\sim 0.45$ \\
\hline $\bar{k}=75, m=9$ & 0.04 & {$[0.045 ; 0.05]$} \\
$\bar{k}=75, m=15$ & 0.11 & {$[0.11 ; 0.115]$} \\
$\bar{k}=75, m=30$ & 0.29 & {$[0.31 ; 0.32]$} \\
$\bar{k}=75, m=45$ & 0.52 & $\sim 0.54$ \\
\hline
\end{tabular}

Table 2: Comparison between the values of the critical fraction of initially active neurons $f^{*}$ obtained by the IQP or the dynamical simulations for $\eta=0.05$. Interval for the dynamical simulation is given by the "jump" values for the 5 th and 95 th percentiles. For the last row in each $\bar{k}$ set, the dynamical simulation displayed a smooth transition, so the value given is the position of the inflexion point.
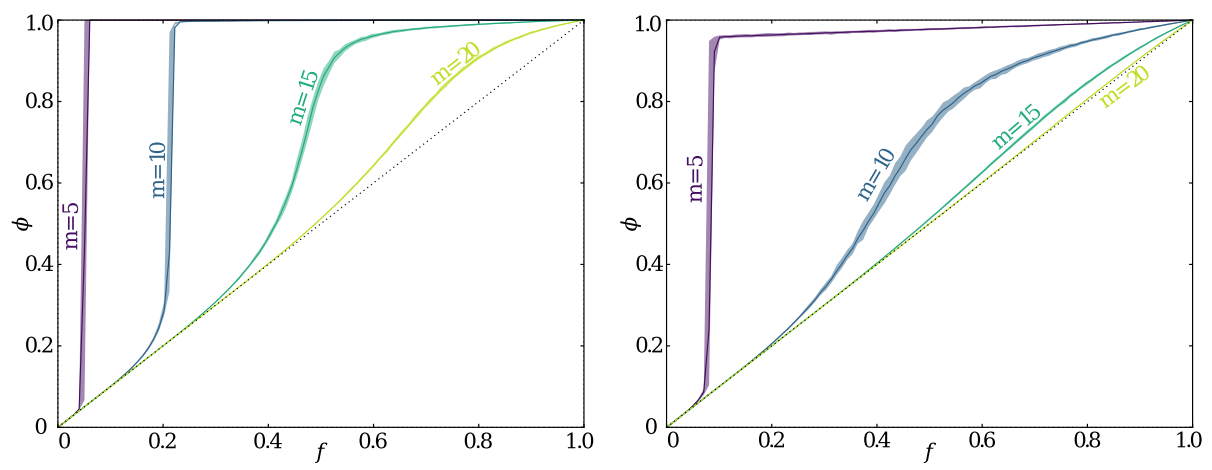

Figure 10: Simulated phase transition for inhibitory fractions of $5 \%$ (left) and 25\% (right) - averaged over 50 runs for each curve to quantify the fluctuations. For the simulations, 10000 neuron networks were generated with Gaussian in-degree distributions $(\bar{k}=25$ and $\sigma_{k}=5$ ). The average transition curve is represented by the solid lines (with increasing quorums $\{5,10,15,20\}$ from dark purple to light green) and the filled area is delimited by the 5 th and 95 th percentiles, i.e. it contains $90 \%$ of the simulated datapoints. The dashed line marks the $\Phi=f$ curve. As in the mean-field model, increasing the inhibitory fraction leads to a sharp decrease in the critical quorum value: $10<m_{c}<15$ for $\eta=0.05$ whereas $5<m_{c}<10$ for $\eta=0.25$. 
In the dynamical simulations, the quorum $m$ was evaluated as the number of simultaneous spikes necessary to make a neuron fire (see Appendix for a more detailed explanation on how its precise value is obtained). The resulting activity of the total population can be seen on Fig. 10. Comparison with Fig. 3 and Fig. 4 shows significant resemblance in the qualitative, as well as in the quantitative 335 behavior of the phase transition. As for the mean-field model, an increase in the fraction $\eta$ of inhibitory neurons leads to a decrease of both the size $g$ of the jump and the final fraction of active neurons $\Phi$. The tendency for the critical value of the quorum to be lower in the dynamical simulations can be easily explained by the combination of the leak conductance and the PSC decrease over time, as detailed in a previous percolation model including decay [19. Beside this small offset, the excellent agreement of the positions where the jump occurs, detailed in Table 2, confirms that the simple IQP percolation model captures the behavior of a more sophisticated dynamical model and is thus relevant to describe the ignition of a burst of activity in a network of coupled neurons.

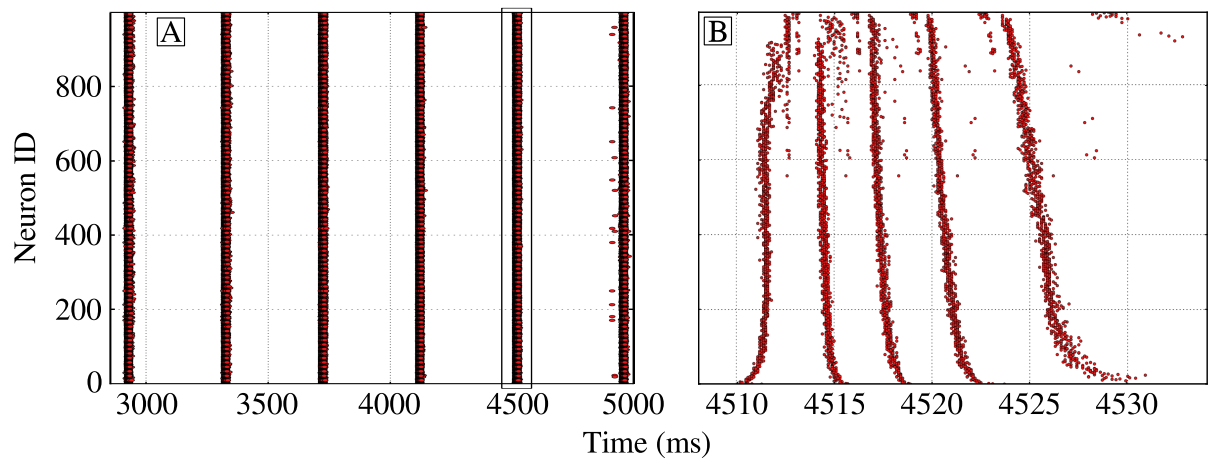

Figure 11: A. Spike raster of a 1000-neuron network with Gaussian in-degree $\mathcal{N}(100,5)$ displaying a spontaneous and periodic bursting behavior. B. shows the inset of the left raster with the detailed dynamics of the successive SBSs. Neurons ordered by increasing in-degree.

Eventually, as can be seen on Fig. 11, the percolation paradigm is perfectly relevant to describe some of the successive network events that occur inside a network burst, for spontaneously active neural networks. Spontaneous bursting activity is a common phenomenon in neuronal networks [27, 15] and the IQP 
can therefore be a useful tool to investigate the properties of this spontaneous behavior.

On Fig. 11 A, the simulated activity is composed of periodic bursts which themselves present a precise substructure as a succession of synchronous burst slices (SBS), shown on 11 B. These are the basic activity blocks that can be described through the percolation formalism. Indeed, after the first spontaneous slice, each subsequent SBS is triggered be the previous one. Because the excitability of the neurons decreases as the burst progresses, this corresponds to a succession of percolation events with increasing values of the quorum. After the last SBS, the value of the quorum becomes greater than $m_{c}$ so no new percolation can occur and the burst terminates.

\section{CONCLUSION}

In this paper, we set out an extension of the Quorum Percolation model with a Gaussian distribution of incoming links (QP) including a fraction $\eta$ of inhibitory neurons (the IQP model). Furthermore, we showed how the mean stationary activation of bursts in a network with inhibitory neurons can be mapped onto an equivalent purely excitatory network endowing an appropriate and different wiring. We provided a quantification of the agreement between the QP and IQP approaches and showed that the agreement is good in usual neuronal cultures, where $\eta \lesssim 20 \%$. Thus, on the issue of large scale response of quorum percolation, mixed inhibitory and excitatory Gaussian random networks with mean input connectivity $\bar{k}$ and fraction $\eta$ of inhibitory neurons, have a purely excitatory Gaussian random network equivalent with a mean incoming links connectivity $(1-2 \eta) \bar{k}$. This enabled us to calculate the critical point of the IQP model as a function of $\eta$. Lastly, we gathered together the approaches coming from the fields of percolation theory and dynamical systems in order to check how the percolation paradigm remains meaningful for the interpretation of a network response to excitation in a biologically more realistic model taking time explicitely into account. We built indeed a dynamical version of the IQP 
model using Brette-Gestner adaptative exponential neurons and alpha-shaped synapses. We showed that Quorum Percolation occurs also in the more sophisticated dynamical framework so that, despite their apparent simplicity, QP and IQP models are an appropriate approach for bursts onset in neuronal cultures.

\section{Appendix}

All dynamical simulations were performed using the NEST simulator 6] with the aeif_psc_alpha model (present on the master branch of the GitHub repository or in release versions strictly higher than 2.11.0) and static synapses.

The neurons were set to adaptive spiking using the neuronal and synaptic parameters detailed in Table 3 .

\begin{tabular}{lccccccccc}
\hline Neuronal parameter & $C_{m}$ & $g_{L}$ & $E_{L}$ & $V_{t h}$ & $I_{e}$ & $\Delta_{T}$ & $a$ & $\tau_{w}$ & $t_{\text {ref }}$ \\
Value & 200 & 9 & -60 & -50 & 0 & 2 & 2 & 600 & 100 \\
\hline Synaptic parameter & $\tau_{s, \text { exc }}$ & $\tau_{s, i n h}$ & $d$ & & & & & & \\
Value & 0.2 & 0.2 & 0.1 & & & & & & \\
\hline
\end{tabular}

Table 3: Neuronal and synaptic parameters used in the simulations. The units are as follow: capacitance in $p F$, conductance in $n S$, voltage in $m V$, current in $p A$ and time in $m s . d$ is the spike transmission delay.

In order to obtain a desired quorum $m$, the synaptic strength between neurons was tuned according to the following procedure:

390

- Send $m$ spikes, each with strength $s$, on a neuron, and increase $s$ until the post-synaptic neuron fires, which occurs for a synaptic strength $s_{m}^{*}$.

- Repeat the process for $m-1$ spikes; this results in a second value $s_{m-1}^{*}$.

- use the synaptic strength $s_{m}=\frac{s_{m}^{*}+s_{m-1}^{*}}{2}$ for all connections in the network. This value of the synaptic strength is important if we want to compare quantita395 tively the predictions of the mean-field model to the simulations. Indeed, in the simulations, the evolution of the state $V_{i}$ of neuron $i$ is progressive, and a spike 
is not necessarily triggered immediately after the excitation. More precisely, at the critical value $s_{m}^{*}$ for which the neuron starts spiking when it receives $m$ spikes, the emission of this spike can take an infinite amount of time (critical slowing down). The choice of $s_{m}$ as the average value between $s_{m}^{*}$ and $s_{m-1}^{*}$ is therefore important to ensures that the neuron will fire rapidly enough (with a characteristic timescale $\tau_{s}$ ) after the reception of $m$ spikes, and thus be in a situation which is comparable to that of the mean-field model.

The networks were generated using the nngt library using the igraph backend.

\section{ACKNOWLEDGEMENTS}

This work was granted access to HPC resources of IDRIS (Orsay) under the allocation 2016057701 made by GENCI (Grand équipement national de calcul intensif).

[1] D. Purves, Neuroscience, Oxford University Press, 2012. URL https://books .google.com/books?id=B5YXRAAACAAJ

[2] J. P. Eckmann, O. Feinerman, L. Gruendlinger, E. Moses, J. Soriano, T. Tlusty, The physics of living neural networks, Physics Reports 449 (1-3) (2007) 54-76. arXiv:1007.5465, doi:10.1016/j . physrep.2007.02.014

[3] E. M. Izhikevich, Dynamical systems in neuroscience, 2007.

URL http://mitpress.mit.edu/item.asp?
ttype $=2\{\&\}$ tid $=11063\{\&\}$ mlid $=600\{\%\} 5$ Cnpapers $: / /$ e5fa1263-0213-4cdb-b4d1-93aaa9302477/Paper/p2818

[4] S. N. Dorogovtsev, a. V. Goltsev, J. F. F. Mendes, Critical phenomena in complex networks, Reviews of Modern Physics 80 (4) (2008) 1275-1335. arXiv:0705.0010, doi:10.1103/RevModPhys.80.1275.

[5] H. Markram, E. Muller, S. Ramaswamy, M. W. Reimann, M. Abdellah, C. A. Sanchez, A. Ailamaki, L. Alonso-Nanclares, N. Antille, S. Arsever, 
G. A. A. Kahou, T. K. Berger, A. Bilgili, N. Buncic, A. Chalimourda, G. Chindemi, J. D. Courcol, F. Delalondre, V. Delattre, S. Druckmann, R. Dumusc, J. Dynes, S. Eilemann, E. Gal, M. E. Gevaert, J. P. Ghobril, A. Gidon, J. W. Graham, A. Gupta, V. Haenel, E. Hay, T. Heinis, J. B. Hernando, M. Hines, L. Kanari, D. Keller, J. Kenyon, G. Khazen, Y. Kim, J. G. King, Z. Kisvarday, P. Kumbhar, S. Lasserre, J. V. Le Bé, B. R. C. Magalhães, A. Merchán-Pérez, J. Meystre, B. R. Morrice, J. Muller, A. MuñozCéspedes, S. Muralidhar, K. Muthurasa, D. Nachbaur, T. H. Newton, M. Nolte, A. Ovcharenko, J. Palacios, L. Pastor, R. Perin, R. Ranjan, I. Riachi, J. R. Rodr \’\iguez, J. L. Riquelme, C. Rössert, K. Sfyrakis, Y. Shi, J. C. Shillcock, G. Silberberg, R. Silva, F. Tauheed, M. Telefont, M. Toledo-Rodriguez, T. Tränkler, W. Van Geit, J. V. D $\backslash$ ’’iaz, R. Walker, Y. Wang, S. M. Zaninetta, J. Defelipe, S. L. Hill, I. Segev, F. Schürmann, Reconstruction and Simulation of Neocortical Microcircuitry, Cell 163 (2) (2015) 456-492. doi:10.1016/j.cell.2015.09.029.

[6] M.-O. Gewaltig, M. Diesmann, NEST (NEural Simulation Tool), Scholarpedia 2 (4) (2007) 1430.

q [7] S. Herculano-Houzel, The Human Advantage: A New Understanding of How Our Brain Became Remarkable, MIT Press, 2016. URL https://books . google.com/books?id=XkzECwAAQBAJ

[8] M. E. Raichle, A brief history of human brain mapping, Trends in Neurosciences 32 (2) (2009) 118-126. doi:10.1016/j.tins.2008.11.001.

[9] P. Guevara, D. Duclap, C. Poupon, L. Marrakchi-Kacem, P. Fillard, D. Le Bihan, M. Leboyer, J. Houenou, J. F. Mangin, Automatic fiber bundle प segmentation in massive tractography datasets using a multi-subject bundle

1. atlas, NeuroImage 61 (4) (2012) 1083-1099. doi:10.1016/j.neuroimage. 2012.02 .071

URL http://dx.doi.org/10.1016/j.neuroimage.2012.02.071

[10] L. Millet, M. U. Gillette, Over a Century of neuron culture: From the 
Hanging drop to Microfluidic devices, Yale Journal of biology and medicine 4 (85) (2012) 500.

[16] O. Cohen, A. Keselman, E. Moses, J. Soriano, T. Tlusty, Quorum perco475 lation in living neural networks 89 (January) (2010) 1-6. doi:10.1209/ 0295-5075/89/18008. 2.0

[17] V. S. Sohal, J. R. Huguenard, Inhibitory interconnections control burst 
23 (26) (2003) 8978-8988. doi:23/26/8978[pii].

$14523100\{\%$ SCnhttp://www.ncbi.nlm.nih.gov/pubmed/14523100

[18] F. Lombardi, H. J. Herrmann, D. Plenz, L. De Arcangelis, On the temporal organization of neuronal avalanches., Frontiers in systems neuroscience 8 (October) (2014) 204. doi:10.3389/fnsys.2014.00204.

[20] P. Monceau, R. Renault, S. Métens, S. Bottani, Effect of threshold disorder on the quorum percolation model, Physical Review E 94 (1) (2016) 1-8. doi:10.1103/PhysRevE.94.012316

[21] S. Métens, P. Monceau, R. Renault, S. Bottani, Finite-size effects and a dynamics of giant transition of a continuum quorum percolation model on 500 random networks, Physical Review E 93 (3) (2016) 032112. doi:10.1103/ PhysRevE.93.032112

URL http://link.aps.org/doi/10.1103/PhysRevE.93.032112

[22] R. Brette, W. Gerstner, Adaptive exponential integrate-and-fire model as an effective description of neuronal activity, J. Neurophysiol. 94 (5) (2005) 3637-3642. doi:10.1152/jn.00686.2005

URL http://www.ncbi.nlm.nih.gov/pubmed/16014787

[23] S. Song, P. J. Sjöström, M. Reigl, S. Nelson, D. B. Chklovskii, Highly nonrandom features of synaptic connectivity in local cortical circuits, PLoS Biology 3 (3) (2005) 507-519. doi:10.1371/journal.pbio.0030068 
[24] D. Stauffer, A. Aharony, Introduction to percolation theory, 1994.

[25] R. Renault, P. Monceau, S. Bottani, S. Métens, Effective non-universality

n. of the quorum percolation model on directed graphs with Gaussian in-degree, Physica A: Statistical Mechanics and its Applications 414 (m) (2014) 352-359. doi:10.1016/j.physa.2014.07.028. S0378437114005974

[26] T. Tlusty, J. P. Eckmann, Remarks on Bootstrap Percolation in Metric Networks, JOURNAL OF PHYSICS A: MATHEMATICAL AND THEORETICAL 42 (2009) 1-11. arXiv:0902.3384, doi:10.1088/1751-8113/ $42 / 20 / 205004$

URL http://arxiv.org/abs/0902.3384

[27] J. G. Orlandi, J. Soriano, E. Alvarez-Lacalle, S. Teller, J. Casademunt, Noise focusing and the emergence of coherent activity in neuronal cultures, Nature Physics 9 (9) (2013) 582-590. doi:10.1038/nphys2686 URL http://dx.doi.org/10.1038/nphys2686http://www.nature.com/ doifinder/10.1038/nphys2686

[28] L. Hernández-Navarro, J. G. Orlandi, B. Cerruti, E. Vives, J. Soriano, Dominance of Metric Correlations in Two-Dimensional Neuronal Cultures Described through a Random Field Ising Model, Physical Review Letters 118 (20) (2017) 1-5. doi:10.1103/PhysRevLett.118.208101. 